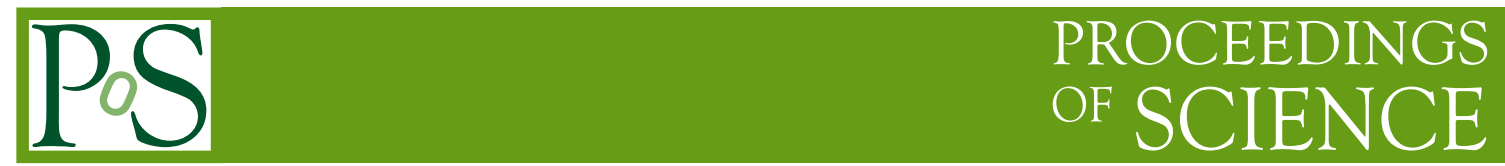

\title{
Strong Interactions - Experimental Overview
}

\author{
Halina Abramowicz ${ }^{* \dagger}$ \\ Tel Aviv University \\ E-mail: halina@post.tau.ac.il
}

\begin{abstract}
Strong interactions, in the perturbative and non-perturbative regime of QCD, play a crucial role in the interpretation of data coming from the hadron colliders experiments. Conversely, data from hadronic interactions provide valuable input for the understanding of QCD dynamics. In this short article, an overview of the latest trends in the strong interactions studies is presented.
\end{abstract}

38th International Conference on High Energy Physics

3-10 August 2016

Chicago, USA

\footnotetext{
* Speaker.

${ }^{\dagger}$ Partly supported by the Israel Science Foundation and the I-CORE Program No. 1937/12.
} 


\section{Introduction}

The theory of strong interactions, Quantum Chromodynamics(QCD), is a well established theory. However, there are limitations in the predictability of QCD calculations both at the low and high interaction scales. Confrontation with measurements is one of the ways to establish the rich dynamics of strong interactions which encompasses confinement, the partonic structure of hadrons and the origin of their masses, as well as the formation of dense matter in the limit of high energy or high temperature.

In the following, a short summary of the advancement in QCD measurements discussed at this conference is presented, starting from soft interactions and ending with hard interactions. It is not meant to be a comprehensive or complete experimental review of strong interactions in hadron collisions and of the available literature on the subject, it is rather meant to reflect the trends while trying the acknowledge the important contribution of all experiments in the field

\section{Cross sections in $p p$ interactions}

One of the first measurements to be performed at new center-of-mass energies, $\sqrt{s}$, is that of the inelastic $p p$ cross section which cannot be calculated from first principles. At the new LHC energy frontier of $13 \mathrm{TeV}$, both ATLAS [1] and CMS [2] measured the inelastic $p p$ cross section, $\sigma_{\text {inel }}$. Due to limited pseudo-rapidity $\eta$ coverage of the detectors, the direct measurements are only sensitive to sufficiently high diffractive masses, $M_{X}$ and $M_{Y}$, where $X$ and $Y$ denote the two proton beam directions. For the ATLAS experiment, which uses the minimum bias scintillator counters covering the range $2.07<|\eta|<3.86, M_{X, Y}>13 \mathrm{GeV}$. In the CMS experiment, the measurements are performed with the forward hadron calorimeters covering $3.0<|\eta|<5.2$ and the CASTOR detector in the range $-6.6<\eta<-5.2$, leading to $M_{X}>4.1 \mathrm{GeV}$ and $M_{Y}>13 \mathrm{GeV}$. Both experiments used MC generators to extrapolate to the full inelastic phase space. The results are as follows:

$$
\begin{array}{ll}
\text { ATLAS } & 78.1 \pm 0.6(\exp ) \pm 1.3(\text { lum }) \pm 2.6(\text { ext }) \mathrm{mb} \\
\text { CMS } & 71.3 \pm 0.5(\exp ) \pm 2.1(\text { lum }) \pm 2.7(\text { ext }) \mathrm{mb}
\end{array}
$$

where the second uncertainty is due to luminosity and the third one is an estimate of the uncertainty due to extrapolation. Assuming that the uncertainties of the two experiments are uncorrelated, the results agree to within $2 \sigma$. Both also agree within uncertainties with various existing parametrizations, though the CMS result tends to be on the low side.

The elastic and the total $p p$ interaction cross sections at the LHC are best determined by measuring the four-momentum transferred squared, $t$, distribution of elastically scattered protons in dedicated very forward detectors. The optical theorem relates the forward elastic scattering cross section to the total cross section by

$$
\sigma_{\mathrm{tot}}^{2}=\left.\frac{16 \pi(\hbar c)^{2}}{1+\rho^{2}} \frac{d \sigma_{\mathrm{el}}}{d t}\right|_{t \rightarrow 0}
$$

This equation can then be used to determine the elastic cross section,

$$
\sigma_{\mathrm{el}}=\frac{\sigma_{\mathrm{tot}}^{2}}{B} \frac{1+\rho^{2}}{16 \pi(\hbar c)^{2}}
$$


where $B$ is the slope of the the $t$ distribution parametrized through $e^{B t}$. The ATLAS Collaboration has recently added a new measurement of $p p$ elastic and total cross section measurements at $\sqrt{s}=$ $8 \mathrm{TeV}$ [3]. The existing measurements of the total, inelastic, elastic, single-diffractive and doublediffractive dissociation cross section for $p p$ and $p \bar{p}$ interactions are compiled in Fig. 1, including cosmic ray measurements. The measurements performed at the LHC fall perfectly in line with expectations based on various parametrizations of the lower energy measurements.
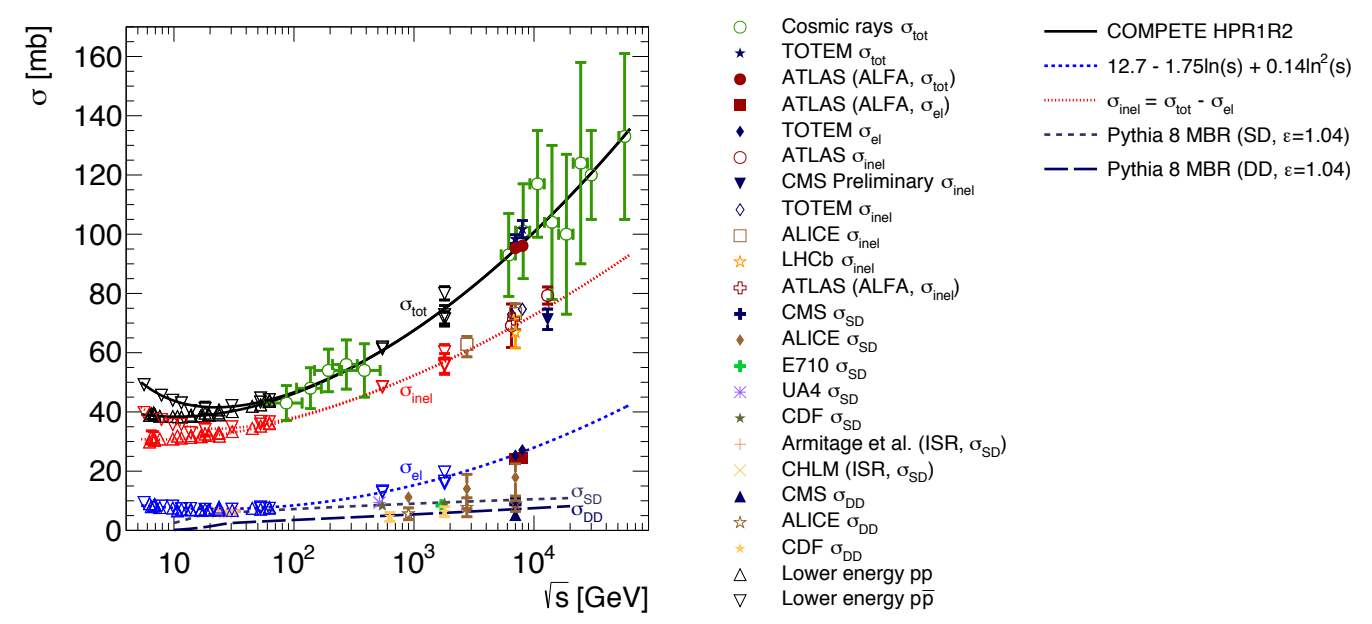

Figure 1: Total $\sigma_{\mathrm{tot}}$, inelastic $\sigma_{\mathrm{inel}}$, elastic $\sigma_{\mathrm{el}}$, single diffractive $\sigma_{\mathrm{SD}}$ and double-diffractive $\sigma_{\mathrm{DD}}$ cross sections as a function of center-of-mass energy $\sqrt{s}$ for $p p$ and $p \bar{p}$ interactions as described in the legend. Overlayed are various parametrizations aslo described in the legend.

\section{Exclusive processes and the Pomeron}

Exclusive processes are understood as the ones in which the beam and target particles remain intact after the interaction and the accompanying final state consists of a well defined color-singlet state such as, for example, two pions or decay products of a heavy vector meson. Such interactions have been and still are intensely studied in deep inelastic $e p$ scattering in order to understand the low- $x$ gluon dynamics, the structure of the Pomeron (IP) and/or the three dimensional structure of the proton (for a discussion see for example [4] and [5]). The same processes can be also studied in $p p$ interactions as the protons are not only a source of a IP flux but also of photons. In particular, the exclusive production of heavy vector mesons in $p p$ interactions at the LHC has been advocated as a potential way to constrain the low- $x$ gluon content of the proton [6]. The LHCb experiment presented measurements of exclusive $J / \psi$ and exclusive $\psi(2 S)$ production at 7 and $13 \mathrm{TeV}$ as a function of the $\gamma^{*} \mathbb{P}$ invariant mass $W$ [7]. The $\gamma p \rightarrow J / \psi p$ cross section as a function of $W$ is shown in Fig. 2, compared to earlier measurements of the H1 and ZEUS experiments. These measurements extend the $W$ reach by an order of magnitude compared to the measurements performed at HERA. For the exclusive $J / \psi$ production, the results agree with the predictions of the NLO calculations based on the $\gamma \mathbb{P}$ fusion in which the Pomeron consists of two gluons [6], while a power-law behavior constrained by the $\mathrm{H} 1$ data [8] predicts too high a cross section. The ratio of $\psi(2 S)$ to $J / \psi$ production cross sections, inferred from the LHCb measurements, seems 
to be $W$ independent contrary to the trend observed in the HERA data [9] of this ratio increasing with $W$. The exclusive production of $\pi^{+} \pi^{-}$investigated by the CMS experiment in $p p$ interactions
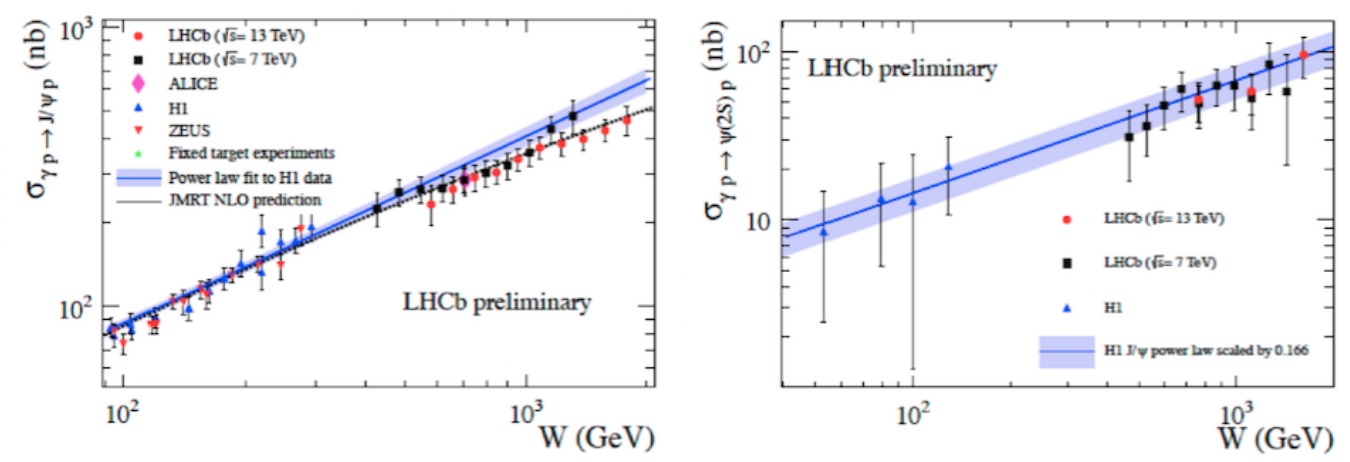

Figure 2: Exclusive $J / \psi$ (left) and $\psi(2 S)$ (right) production cross section in $\gamma p$ interactions derived by the LHCb experiment [7] from $p p$ scattering as a function of the $\gamma p$ center of mass energy $W$ compared to other measurements as described in the legend.

at $7 \mathrm{TeV}$ indicates not only the presence of $\gamma \mathrm{IP}$ fusion into a $\rho$ meson in the distribution of the invariant mass of the $\pi^{+} \pi^{-}$system but also the presence of the $f_{0}$ and $f_{2}$ mesons as expected from double-IP exchange [10]. Exclusive production of $\pi^{+} \pi^{-}$in ep interactions accompanied by a forward scattered neutron can be interpreted in terms of $\gamma \pi$ scattering. The $\gamma \pi$ scattering cross section extracted by the $\mathrm{H} 1$ experiment [11] comes out to be only half of the value expected from the additive quark model and the value of $\gamma p$ cross section, indicating the presence of strong absorptive corrections.

\section{Event characteristics - forward physics}

The spectra of forward-produced neutrons [12] and $\pi^{0}$ [13] have been investigated by the LHCf Collaboration, whose calorimeters are located at $\pm 140 \mathrm{~m}$ from the ATLAS interaction point. The aim of this experiment is the study at the LHC accelerator of the neutral-particle production cross sections in the very forward region of proton-proton and nucleus-nucleus interactions to provide essential input for understanding the development of atmospheric showers induced by very high energy cosmic rays hitting the Earth atmosphere. As shown in Fig. 3 the energy distribution for forward neutrons measured in $p p$ interactions at $7 \mathrm{TeV}$ cannot be reproduced by any of the available MC generators. The difficulty in obtaining a proper description of the forward energy flow has been also observed by the CMS experiment which compared the total energy measured in the range $-6.6<\eta<-5.5$ in $p p$ interactions at $13 \mathrm{TeV}$ to various MC generators [14]. Moreover, while the data taken at different $\sqrt{s}$ at the LHC indicate that Feynman scaling holds in the forward region $[13,15]$, in the MC generators the scaling is pushed to the extreme forward region. This is shown in Fig. 3 where the transverse energy as a function of pseudo-rapidity measured with respect to the rapidity of the beam protons, $\eta-y_{\text {beam }}$, is shown for various $\sqrt{s}$ and compared to MC expectations in generators typically used in cosmic-ray physics. As more data become available, this issue should attract the attention of model builders as it may affect the precision measurements at the LHC. 

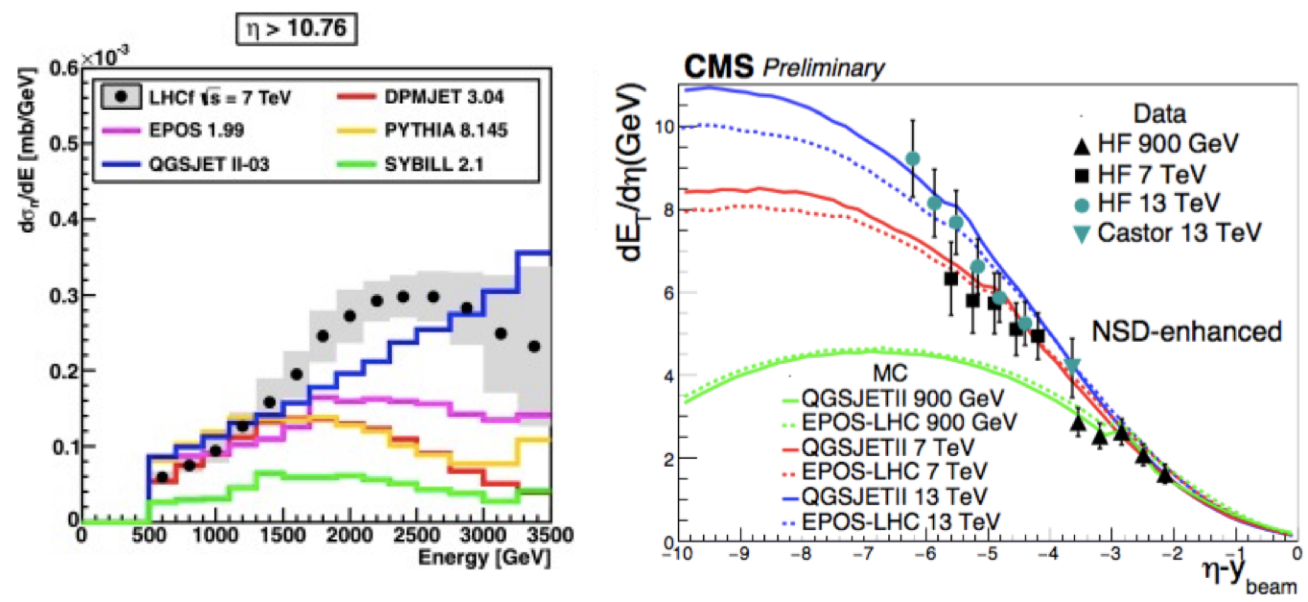

Figure 3: Left - energy distribution of forward produced neutrons in $p p$ interactions as measured by the LHCf experiment at $7 \mathrm{TeV}$ [12], compared to various model expectations as described in the legend. Right transverse energy $E_{T}$ as a function of pseudo-rapidity measured with respect to the rapidity of beam protons $\eta-y_{\text {beam }}$ by the CMS experiment [14] for $p p$ interactions at different center-of-mass energies, compared to various MC generators, as explained in the legend.

\section{Event characteristics - central production}

The central production region at the LHC is still not fully under control. In spite of a large effort to tune the MC generators to describe properly the event characteristics of $p p$ interactions at $\sqrt{s}$ of 7 and $8 \mathrm{TeV}$, further tuning is necessary for $13 \mathrm{TeV}[16,17]$. This is illustrated in Fig. 4 where the transverse momentum $p_{T}$, multiplicity $n_{c h}$ and pseudo-rapidity $\eta$ distributions of charged particles is compared to various MC generators. Especially in the tails of the $p_{T}$ and $n_{c h}$ distributions, the MC may miss the data by as much as $50 \%$. This is also reflected in the underlying event characteristics illustrated in Fig. 4 through the comparison of data and MC expectations, at various center of mass energies, of the behavior of the average total transverse momentum of charged particles with $|\eta|<2$ and $p_{T}>0.5 \mathrm{GeV}$, in the azimuthal region transverse to the direction of the leading jet (of charged particles) in the event, as a function of the $p_{T}$ of that jet. There are also unexpected effects in high energy $p p$ interactions at the LHC, the dynamic origin of which is not yet established. Already in the $7 \mathrm{TeV} p p$ interaction data, CMS [18] established that for large multiplicities $\left(n_{c h}>100\right)$, a long-range structure in the two-particle angular correlations functions in the $\eta$ and azimuthal angle $\phi$ appears on the same side as the leading transverse momentum particle. While such a structure is expected on the away-side to compensate the transverse momentum, the appearance of a ridge on the near-side is rather typical of heavy-ion collisions (for a discussion see [19]). The presence of this long-range near-side correlations in $p p$ interactions with large multiplicities has been now also established at $13 \mathrm{TeV}[20,21]$ as shown in Fig. 5. The other interesting effect related to charged particle multiplicity is the increase of strange particles production with increasing charged multiplicity, the stronger the heavier the particle, observed by ALICE [22] and shown in Fig. 5. The increase with multiplicity follows the same trend as observed in $p \mathrm{~Pb}$ interactions. The standard MC generator used to model minimum-bias $p p$ events, PYTHIA8 [23], predicts a constant production rate, dependent on the mass but independent of multiplicity and in general it expects 

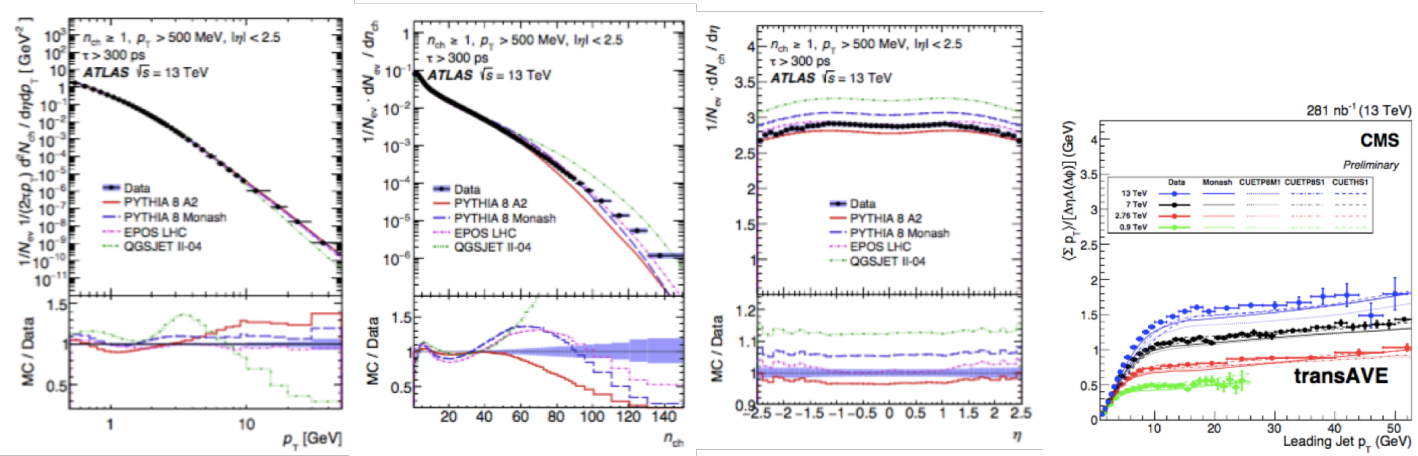

Figure 4: Left - transverse momentum $p_{T}$, charged multiplicity $n_{c h}$ and pseudo-rapitiy $\eta$ distributions measured by the ATLAS experiment [16] in $p p$ interactions at $13 \mathrm{TeV}$. Right - average total transverse momentum in the azimuthal region transverse to the direction of the leading jet (of charged particles) in the event, $<\sum p_{T}>$, as a function of the transverse momentum $p_{T}$ of that jet as measured by the CMS experiment [17] at different center of mass energies of $p p$ interactions. The data are compared to expectations of various MC generators as described in the legend.
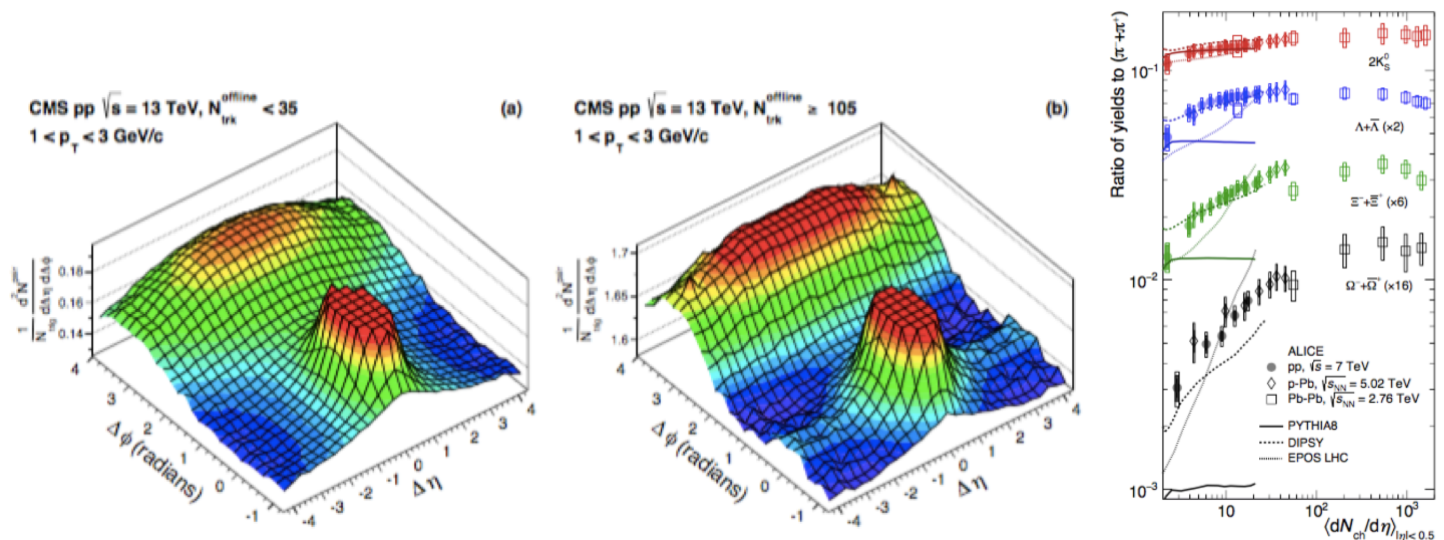

Figure 5: Left - two-particle correlation function as a function of the azimuthal $\Delta \phi$ and pseudo-rapidity $\Delta \eta$ distance between two particles in $p p$ interactions at $13 \mathrm{TeV}$ as measured by the CMS experiment [21] for multiplicity $N_{\text {trk }}^{\text {offline }}$ smaller or bigger-equal to 105 charged particles as described in the legend. Right - rate of production of strange particles relative to the pion production rate as a function of the average multiplicity of centrally produced charged particles $\left.\left\langle d N_{\mathrm{ch}} / d y\right\rangle\right|_{|e t a|<0.5}$ as measured by the ALICE experiment [22] in $p p, p \mathrm{~Pb}$ and $\mathrm{PbPb}$ interactions at different center-of-mass energies, compared to expectations of various MC generators as described in the legend.

rates much lower than observed, especially for strange particles other than the kaons. An increase is expected in the EPOS LHC [24] generator with a rate higher than observed in the data while the novel DIPSY [25] generator, based on a dipole picture of the $p p$ interactions reproduces well the observed trend up to the $\Omega$ production.

\section{Parton distribution functions of the proton}

An important input to modeling $p p$ interactions are the parton distribution functions (PDF) of the proton. The inclusive neutral current and charged current $e^{ \pm} p$ interaction cross section mea- 
sured at HERA constitute an essential anchor for all existing PDF parametrizations. However, the combined H1 and ZEUS measurements [26] provide enough constraints to extract PDFs from these data only. The advantage of such an approach lies in well-understood experimental uncertainties. Recently, the HERA community provided a new family of parametrizations under the name of HERAPDF2.0, both at NLO and at NNLO. The sets provide a scan in the strong coupling constant $\alpha_{\mathrm{S}}$, alternative gluon parametrizations as well as different flavor schemes. There are also sets which are derived with the addition of jet-production as well as charm production cross sections from the combined $\mathrm{H} 1$ and ZEUS data. The quality of the fits is shown in Fig. 6 where the NC and CC reduced cross sections as a function of the scale $Q^{2}$ for $e^{ \pm} p$ are compared to NLO (NC) and NNLO (CC) HERAPDF2.0 parametrization at selected values of Bjorken- $x$. .
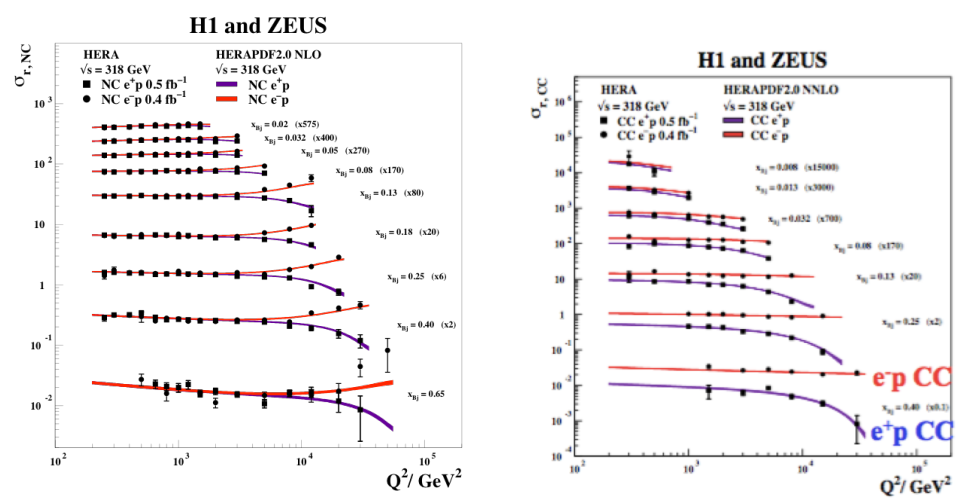

Figure 6: Neutral current (left) and charged-current (right) $e^{ \pm} p$ cross sections as a function of $Q^{2}$ for selected values of Bjorken- $x$, compared to NLO (NC) and NNLO (CC) HERAPDF2.0 PDF parametrizations [26].

\section{Multi-parton interactions}

Multi parton-parton interactions (MPIs) within the same $p p$ interaction play an important role in modeling the underlying event activity and the minimum bias $p p$ interactions. They are mainly driven by the large flux of low- $x$ partons, however, at the LHC energies they may easily be a competing source of hard final states. From purely geometrical considerations, MPIs are a potential source of information about the three-dimensional structure of the proton and parton-parton correlations. The study of these interactions, and in particular of double-parton scattering (DPS), is still in its infancy, though the accumulated measurements from the Tevatron and the LHC have attracted a lot of theoretical attention [27].

In the absence of a detailed theoretical framework, the DPS results are usually interpreted in terms of a pocket formula, in terms of an effective cross section, $\sigma_{\text {eff }}$, as in

$$
\sigma_{\mathrm{DPS}}^{A B}=\frac{m}{2} \frac{\sigma_{\mathrm{SPS}}^{A} \sigma_{\mathrm{SPS}}^{B}}{\sigma_{\mathrm{eff}}}
$$

where SPS and DPS denote single- and double-parton scattering processes and the combinatorial factor $m=1$ for identical final states $A$ and $B$ and $m=2$ for $A \neq B$. This simple formula is derived 
under the assumption of independent parton scatterings, with

$$
\sigma_{\mathrm{eff}}=\left[\int d^{2} b T^{2}(\mathbf{b})\right]^{-1},
$$

where $T(\mathbf{b})$ is the overlap function that characterizes the transverse area occupied by the interacting partons in the impact parameter space $\mathbf{b}$. The smaller the $\sigma_{\text {eff }}$, the higher the local parton density and the larger the probability of DPS to occur. If indeed the assumption of factorization is correct, the value of $\sigma_{\text {eff }}$ should be universal, independent of final states or phase space.

Experimentally, the determination of $\sigma_{\text {eff }}$ is based on the percentage of identified DPS events. The latter is extracted based on topological considerations which involves a proper understanding of topologies expected in SPS. At this conference results were presented by the D0 experiment in the $2 \gamma+2$ jets final states [28], by the CMS experiment in same sign $W$-pair production [29] and by the ATLAS experiment in 4-jets [30] and $J / \psi J / \psi$ [31] final states. A compilation of existing determination of $\sigma_{\text {eff }}$ values or limits is shown in Fig. 7. For most final states, $\sigma_{\text {eff }}$ varies from about

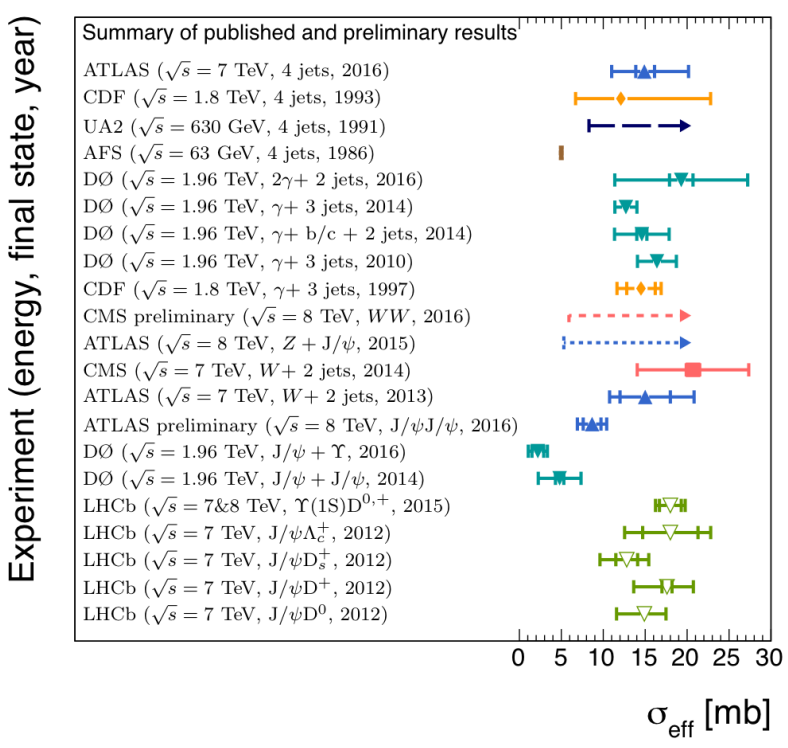

Figure 7: Compilation of existing estimates of the effective cross section $\sigma_{\text {eff }}$ from measurements of doubleparton scattering with the final states and the corresponding experiments listed in the legend.

15 to $20 \mathrm{mb}$ indicating a universal value. The exception are final states consisting of heavy vector mesons, $J / \psi$ and/or $\Upsilon$, for which $\sigma_{\text {eff }}$ is markedly smaller and determined with small uncertainties. The origin of this effect is not yet understood. The interesting observation is that $\sigma_{\text {eff }}$ is smaller than the inelastic $p p$ cross section indicating that the partons (mostly gluons) are located in an area smaller than expected from the charged radius of the proton. It has also been pointed out that the value of $\sigma_{\text {eff }}$ derived from the gluon formfactor of the proton is larger than the one observed in DPS indicating the presence of parton-parton correlations not included in the pocket formula [32].

\section{Perturbative QCD stress tests}

The ability to describe reliably the production of final states in hard interactions is essential 
for all the searches of physics beyond the Standard Model. This is particularly true for multi-leg, multi-jet processes. Because of the nature of the QCD perturbative expansion, data driven studies are extremely important.

As shown by CMS [33], inclusive jet production coss section is very well reproduced by NLO calculations in a wide range of rapidity and transverse momentum. An example is shown in Fig. 8 for $13 \mathrm{TeV} p p$ interactions. These measurements can be used as an additional constraint in extracting PDFs from the HERA data. This results in a substantial improvement of the uncertainties in the determination of the gluon density, in particular for $10^{-3} \lesssim x \lesssim 5 \cdot 10^{-1}$ as shown in Fig. 8. Studies
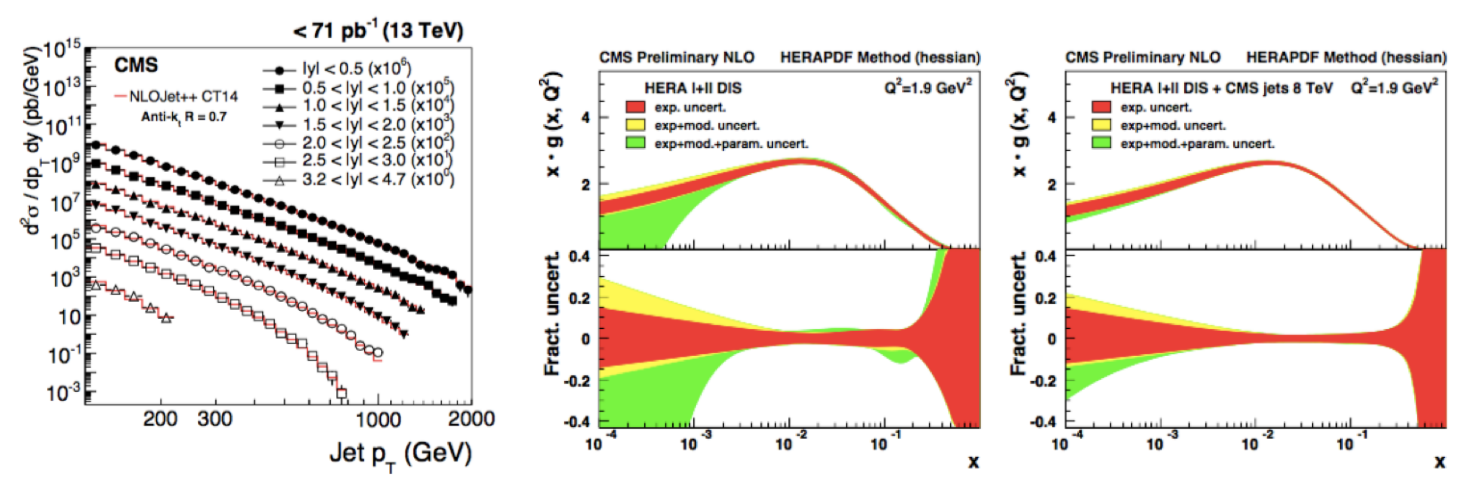

Figure 8: Left - inlusive jet production cross section as a function of jet transverse-momentum $p_{T}$ in different rapidity $y$ ranges measured by the CMS experiment [33] at $13 \mathrm{TeV}$ compared to NLO calculations as explained in the legend. Right - gluon distribution and its uncertainties (explained in the legend) derived from NLO QCD PDF fits to HERA data alone or with the inclusion of inclusive jet cross sections measured in $p p$ interactions at $8 \mathrm{TeV}$ by the CMS experiment [34].

of inclusive prompt-photon production have been presented by ATLAS [35] and CDF [36]. The comparison of the cross section as a function of transverse energy to expectations of NLO QCD calculations is shown in Fig. 9. Here also a very good agreement is observed. The same cannot be
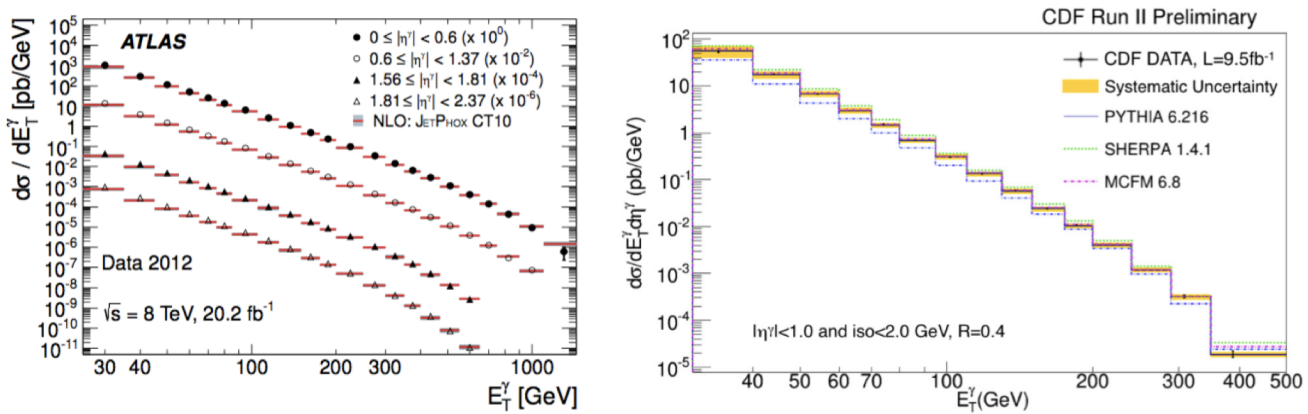

Figure 9: Inclusive prompt-photon production cross section as a function of the photon transverse energy $E_{T}^{\gamma}$ measured in $p p$ collisions by the ATLAS experiment [35] at $8 \mathrm{TeV}$ in different $\gamma$ pseudo-rapidity $\eta^{\gamma}$ ranges and in $p \bar{p}$ collisions at $1.96 \mathrm{TeV}$ measured by the CDF experiment [36] for $\left|\eta^{\gamma}\right|<1$. The measurements are compared to NLO calculations and MC generators as described in the legends.

said about multi-jet production. For dijet production in $e p$ scattering, recently NNLO calculations become available [37] and the comparison to the H1 dijet measurements [38] is shown in Fig. 10. 
The trend observed in the data is better reproduced by the NNLO calculations than the NLO ones, however the overall normalization, in particular at higher jet $p_{T}$, seems to be off. At much higher scales, the ATLAS experiment [39] compared the invariant mass of four jets produced in $p p$ interactions at $8 \mathrm{TeV}$ to expectations of various multi-leg MC generators. Here again there is room for improvement in the calculations as shown in Fig. 10. In general, the lower the multiplicity of jets
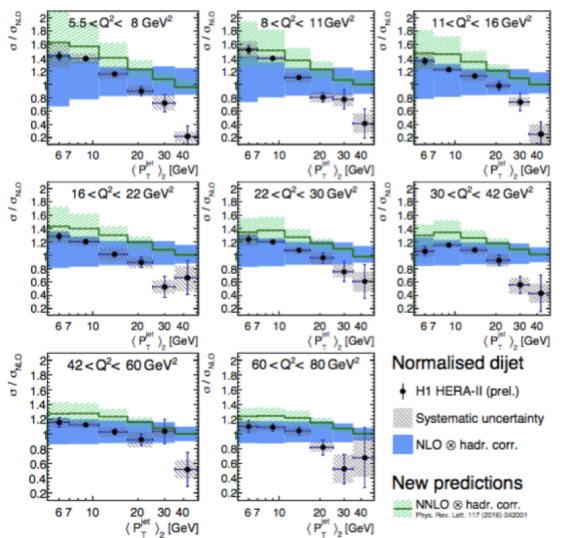
+ H1 HERA-III (prel.)

Systematic uncertainty

New predictions Th: NNLO \& nadr. corr.
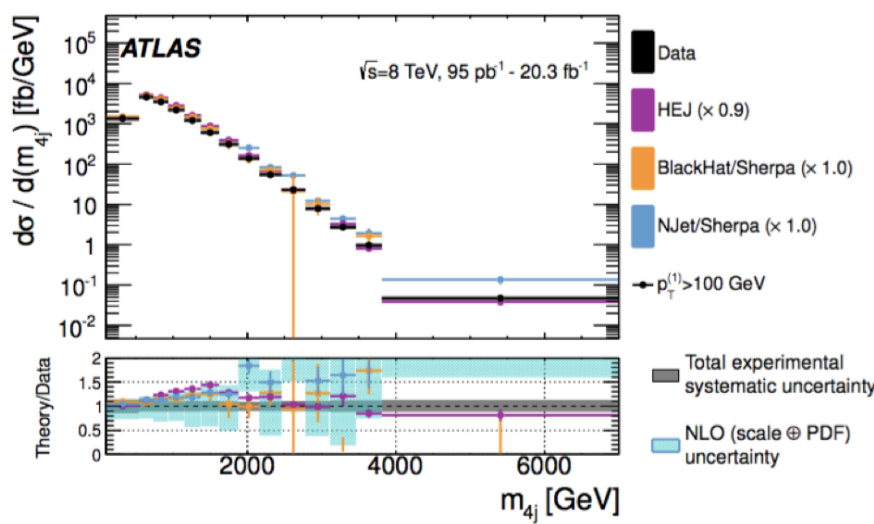

Figure 10: Left - dijet production cross section measured by the $\mathrm{H} 1$ experiment [38] compared to NLO and NNLO calculations as a function of the average transverse momentum of the two jets, $<p_{T}^{\text {jet }}>_{2}$, in terms of cross section ratios. Right - Invariant mass distribution of a system of four jets, $m_{4 \mathrm{j}}$, measured by the ATLAS experiment [39] compared to various MC generators as described in the legend.

the better the description by perturbative QCD. This is shown for the case of $Z$ and $W$ production in association with jets at $13 \mathrm{TeV}$ in Fig. 11 where the cross sections for multi-jet production obtained by ATLAS [40] and CMS [41] are shown as a function of the inclusive jet multiplicity and are compared to various multi-leg MC generators.
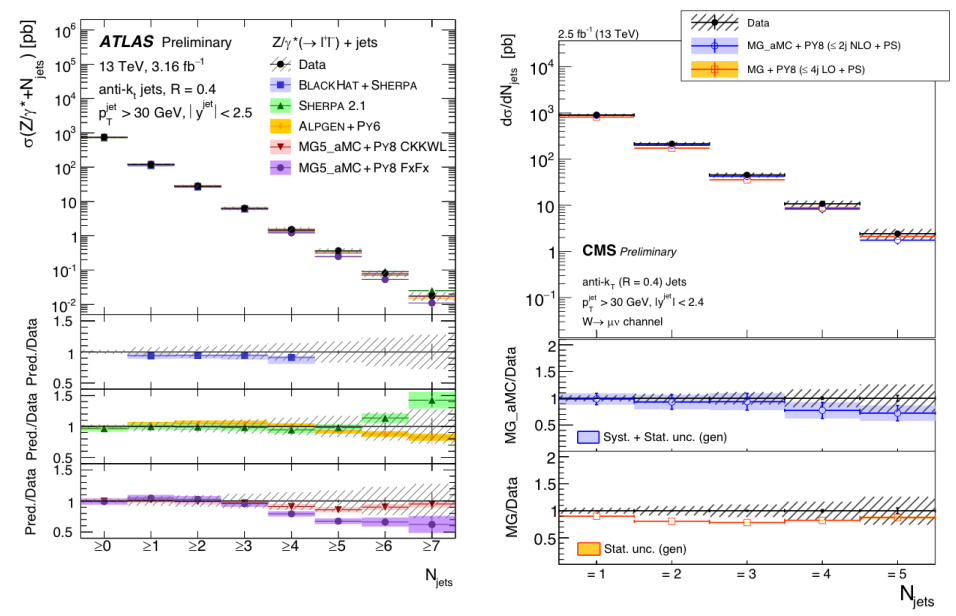

Figure 11: Cross section for jets accompanying the production of $Z / \gamma^{*}$ (left) and $W^{ \pm}$(right) in $p p$ collisions at $13 \mathrm{TeV}$ as a function of the lower limit on the number $N_{\text {jets }}$ of produced jets. The measurements by the ATLAS $\left(Z / \gamma^{*}\right)$ [40] and CMS $\left(W^{ \pm}\right)$[41] experiments are compared to various MC generators as described in the legends. 


\section{Summary}

Given the complexity of final states produced in strong interactions, all in all a good description of the main event characteristics is achieved. However, certain regions of phase space, like the forward region or high multiplicities, are still poorly controlled. The impact of collective effects is not well understood either. The HERA data constitute a anchor for the determination of the parton distributions in the proton but the precision at low- and high- $x$ is limited though it can be substantially improved with additional data. Under the circumstances, the main concern is that the lack of precise Standard Model expectations may hinder searches of physics beyond.

\section{Acknowledgements}

I would like to thank all the Collaborations for their prompt response and invaluable help in preparing this presentation.

\section{References}

[1] ATLAS Collaboration, "Measurement of the Inelastic Proton-Proton Cross Section at $\sqrt{s}=13 \mathrm{TeV}$ with the ATLAS Detector at the LHC," Phys. Rev. Lett. 117, no. 18, 182002 (2016) [arXiv:1606.02625 [hep-ex]].

[2] CMS Collaboration, "Measurement of the inelastic proton-proton cross section at $\sqrt{s}=13 \mathrm{TeV}$," CMS-PAS-FSQ-15-005.

[3] ATLAS Collaboration, "Measurement of the total cross section from elastic scattering in $p p$ collisions at $\sqrt{s}=8 \mathrm{TeV}$ with the ATLAS detector," Phys. Lett. B 761, 158 (2016) [arXiv:1607.06605 [hep-ex]].

[4] S. Wallon, "Hard exclusive processes," Acta Phys. Polon. Supp. 6, 587 (2013) [arXiv:1302.2888 [hep-ph]].

[5] L. Frankfurt, M. Strikman and C. Weiss, "Small-x physics: From HERA to LHC and beyond," Ann. Rev. Nucl. Part. Sci. 55, 403 (2005) [hep-ph/0507286].

[6] S. P. Jones, A. D. Martin, M. G. Ryskin and T. Teubner, "The exclusive $J / \psi$ process at the LHC tamed to probe the low $x$ gluon," Eur. Phys. J. C 76, no. 11, 633 (2016) [arXiv:1610.02272 [hep-ph]].

[7] LHCb Collaboration, "Central exclusive production of $J / \psi$ and $\psi(2 S)$ mesons in $p p$ collisions at $\sqrt{s}=13 \mathrm{TeV}$, LHCb-CONF-2016-007.

[8] C. Alexa et al. [H1 Collaboration], "Elastic and Proton-Dissociative Photoproduction of J/psi Mesons at HERA,” Eur. Phys. J. C 73, no. 6, 2466 (2013) [arXiv:1304.5162 [hep-ex]].

[9] H. Abramowicz et al. [ZEUS Collaboration], "Measurement of the cross-section ratio $\sigma_{\psi(2 S)} / \sigma_{J / \psi(1 S)}$ in deep inelastic exclusive ep scattering at HERA," Nucl. Phys. B 909, 934 (2016) [arXiv:1606.08652 [hep-ex]].

[10] CMS Collaboration, "Measurement of exclusive $\pi^{+} \pi^{-}$production in proton-proton collisions at $\sqrt{s}=7 \mathrm{TeV}$, , CMS-PAS-FSQ-12-004.

[11] V. Andreev et al. [H1 Collaboration], "Exclusive $\rho^{0}$ meson photoproduction with a leading neutron at HERA,” Eur. Phys. J. C 76, no. 1, 41 (2016) [arXiv:1508.03176 [hep-ex]]. 
[12] O. Adriani et al. [LHCf Collaboration], "Measurement of very forward neutron energy spectra for 7 TeV proton-proton collisions at the Large Hadron Collider," Phys. Lett. B 750, 360 (2015) [arXiv:1503.03505 [hep-ex]].

[13] O. Adriani et al. [LHCf Collaboration], "Measurements of longitudinal and transverse momentum distributions for neutral pions in the forward-rapidity region with the LHCf detector," Phys. Rev. D 94, no. 3, 032007 (2016) [arXiv:1507.08764 [hep-ex]].

[14] CMS Collaboration, "Measurement of the energy distribution in the very forward direction at $13 \mathrm{TeV}$ with CMS," CMS-PAS-FSQ-16-002.

[15] CMS Collaboration, "Measurement of the pseudorapidity dependence of the energy and transverse energy density in pp collisions at $\sqrt{s}=13 \mathrm{TeV}$ with CMS," CMS-PAS-FSQ-15-006.

[16] ATLAS Collaboration, "Charged-particle distributions in $\sqrt{s}=13 \mathrm{TeV} p p$ interactions measured with the ATLAS detector at the LHC," Phys. Lett. B 758, 67 (2016) [arXiv:1602.01633 [hep-ex]].

[17] CMS Collaboration, "Underlying Event Measurements with Leading Particles and Jets in pp collisions at $\sqrt{s}=13$ TeV," CMS-PAS-FSQ-15-007.

[18] CMS Collaboration], "Observation of Long-Range Near-Side Angular Correlations in Proton-Proton Collisions at the LHC," JHEP 1009, 091 (2010) [arXiv:1009.4122 [hep-ex]].

[19] Marco Van Leeuwen, "Heavy Ions Overview”, talk presented at this conference.

[20] ATLAS Collaboration, "Observation of Long-Range Elliptic Azimuthal Anisotropies in $\sqrt{s}=13$ and 2.76 TeV pp Collisions with the ATLAS Detector,” Phys. Rev. Lett. 116, no. 17, 172301 (2016) [arXiv:1509.04776 [hep-ex]].

[21] CMS Collaboration, "Measurement of long-range near-side two-particle angular correlations in pp collisions at $\sqrt{s}=13$ TeV," Phys. Rev. Lett. 116, no. 17, 172302 (2016) [arXiv:1510.03068 [nucl-ex]].

[22] J. Adam et al. [ALICE Collaboration], "Multiplicity-dependent enhancement of strange and multi-strange hadron production in proton-proton collisions at $\sqrt{s}=7 \mathrm{TeV}$," arXiv:1606.07424 [nucl-ex].

[23] T. Sjöstrand et al., "An Introduction to PYTHIA 8.2," Comput. Phys. Commun. 191, 159 (2015) [arXiv:1410.3012 [hep-ph]].

[24] T. Pierog, I. Karpenko, J. M. Katzy, E. Yatsenko and K. Werner, "EPOS LHC: Test of collective hadronization with data measured at the CERN Large Hadron Collider," Phys. Rev. C 92, no. 3, 034906 (2015) [arXiv:1306.0121 [hep-ph]].

[25] C. Flensburg, G. Gustafson and L. Lonnblad, "Inclusive and Exclusive Observables from Dipoles in High Energy Collisions,” JHEP 1108, 103 (2011) [arXiv:1103.4321 [hep-ph]].

[26] H. Abramowicz et al. [H1 and ZEUS Collaborations], "Combination of measurements of inclusive deep inelastic $e^{ \pm} p$ scattering cross sections and QCD analysis of HERA data,” Eur. Phys. J. C 75, no. 12, 580 (2015) [arXiv:1506.06042 [hep-ex]].

[27] The interested reader is referred to the series of workshops on multi-parton Interactions, MPI@LHC, as the literature is vast but there is still no comprehensive review of the substantial theoretical progress. The link to the latest workshop is http://www. nucleares. unam.mx/MPI2016/.

[28] V. M. Abazov et al. [D0 Collaboration], "Study of double parton interactions in diphoton + dijet events in $p \bar{p}$ collisions at $\sqrt{s}=1.96$ TeV," Phys. Rev. D 93, no. 5, 052008 (2016) [arXiv:1512.05291 [hep-ex]]. 
[29] CMS Collaboration, "Double Parton Scattering cross section limit from same-sign W bosons pair production in di-muon final state at LHC," CMS-PAS-FSQ-13-001.

[30] ATLAS Collaboration, "Study of hard double-parton scattering in four-jet events in pp collisions at $\sqrt{s}=7 \mathrm{TeV}$ with the ATLAS experiment," JHEP 1611, 110 (2016) [arXiv:1608.01857 [hep-ex]].

[31] ATLAS Collaboration, "Measurement of the prompt $J / \psi$ pair production cross-section in $p p$ collisions at $\sqrt{s}=8 \mathrm{TeV}$ with the ATLAS detector," arXiv:1612.02950 [hep-ex].

[32] L. Frankfurt and M. Strikman, "Two gluon form-factor of the nucleon and $J / \psi$ photoproduction," Phys. Rev. D 66, 031502 (2002)

[33] CMS Collaboration, "Measurement of the double-differential inclusive jet cross section in proton-proton collisions at $\sqrt{s}=13$ TeV," Eur. Phys. J. C 76, no. 8, 451 (2016) [arXiv:1605.04436 [hep-ex]].

[34] CMS Collaboration, "Measurement of the double-differential inclusive jet cross section at $\sqrt{s}=8 \mathrm{TeV}$, , CMS-PAS-SMP-14-001.

[35] ATLAS Collaboration, "Measurement of the inclusive isolated prompt photon cross section in pp collisions at $\sqrt{s}=8 \mathrm{TeV}$ with the ATLAS detector," JHEP 1608, 005 (2016) [arXiv:1605.03495 [hep-ex]].

[36] CDF Collaboration, "Measurement of the Inclusive Isolated Prompt Photon cross section in $p \bar{p}$ collisions at $\sqrt{s}=1.96 \mathrm{TeV}$, suding $9.5 \mathrm{fb}^{-1}$," CDF-note-11180.

[37] J. Currie, T. Gehrmann and J. Niehues, "Precise QCD predictions for the production of dijet final states in deep inelastic scattering,” Phys. Rev. Lett. 117, no. 4, 042001 (2016) [arXiv:1606.03991 [hep-ph]].

[38] H1 Collaboration, "Measurement of Normalised Cross Sections for Jet Production in ep Collisions at HERA and Comparison to Next-to-next-to-leading Order QCD Predictions," H1prelim-16-062

[39] ATLAS Collaboration, "Measurement of four-jet differential cross sections in $\sqrt{s}=8 \mathrm{TeV}$ proton-proton collisions using the ATLAS detector,” JHEP 1512, 105 (2015) [arXiv:1509.07335 [hep-ex]].

[40] ATLAS Collaboration, "Measurements of the Production Cross Section of a $Z$ Boson in Association with Jets in $p p$ collisions at $\sqrt{s}=13 \mathrm{TeV}$ with the ATLAS Detector," ATLAS-CONF-2016-046.

[41] CMS Collaboration, "Measurement of the differential cross section for the production of a W (rightarrow munu) boson in association with jets at sqrt(s) = 13 TeV," CMS-PAS-SMP-16-005. 